\title{
Evaluation of Turbulent Particle Flux by Heavy Ion Beam Probe in the T-10 Tokamak*)
}

\author{
Leonid G. ELISEEV ${ }^{1)}$, Alexander V. MELNIKOV ${ }^{1,2)}$, Sergey E. LYSENKO ${ }^{1)}$, \\ Philipp O. KHABANOV ${ }^{1,4)}$, Vitaly N. ZENIN ${ }^{1,4)}$, Mikhail A. DRABINSKIJ ${ }^{1,4)}$, \\ Nikolaj K. KHARCHEV ${ }^{1)}$, Alexander S. KOZACHEK ${ }^{3)}$, Ludmila I. KRUPNIK ${ }^{3)}$ \\ and HIBP Team ${ }^{3)}$ \\ 1) National Research Centre "Kurchatov Institute”, 123182, Moscow, Russia \\ 2) National Research Nuclear University MEPhI, 115409, Moscow, Russia \\ ${ }^{3)}$ Institute of Plasma Physics, NSC KIPT, 310108, Kharkov, Ukraine \\ 4) Moscow Institute of Physics and Technology, 141700, Dolgoprudny, Russia
}

(Received 27 December 2017 / Accepted 24 May 2018)

\begin{abstract}
In the T-10 tokamak, the local fluctuations of poloidal electric field $\tilde{E}_{\mathrm{pol}}$ and density $\tilde{n}_{\mathrm{e}}$ were simultaneously measured by heavy ion beam probe (HIBP) with 5-slit energy analyzer that allow us to estimate the turbulent particle flux and $E \times B$ rotation velocity in the gradient zone of plasma column $(r / a=0.8)$. It was shown that at the Ohmic heating $(\mathrm{OH})$ stage of discharge, the outward flux is in the range of $2 \times 10^{19} \mathrm{~m}^{-2} \mathrm{~s}^{-1}$, increasing at the ECRH stage by a factor of 1.5 . At $\mathrm{OH}$ stage, the poloidal rotation velocity is about $4.5 \mathrm{~km} / \mathrm{s}$.
\end{abstract}

(C) 2018 The Japan Society of Plasma Science and Nuclear Fusion Research

Keywords: turbulent flux, HIBP, T-10 tokamak, quasicoherent mode

DOI: $10.1585 /$ pfr.13.3402106

\section{Introduction}

Many years of fusion researches have shown that the energy and particle losses are realized through several channels, e.g. via neoclassical losses. On top of that, turbulent losses play an important role. In its turn, the turbulent transport has a complex structure induced by the interplay of fine-scale turbulence (e.g., ITG, TEM and other modes) via middle-scale turbulence (zonal flow, $\mathrm{ZF}$, and geodesic acoustic mode, GAM) and global events (e.g., L-H transitions). Therefore, the direct measurements of the turbulent particle flux presents an interesting and important task. At the plasma edge, the turbulent particle flux may be measured by multipin Langmuir probes, while in the core plasmas it may be measured by multichannel Heavy Ion Beam Probe (HIBP) [1].

HIBP is well known as a unique diagnostics to measure directly plasma electrostatic potential in the core area of toroidal plasmas $[2,3]$. High spatial and temporal resolution of the modern multichannel HIBP makes it an effective tool to study plasma oscillations, both broadband turbulence $[4,5]$ and quasicoherent oscillations like GAMs [6-8] and MHD tearing modes [9]. HIBP was also used to study Alfvén eigenmodes [10-12] including related turbulent particle flux [13].

The radial turbulent particle flux is determined as $\Gamma_{\mathrm{r}}=$ $\left\langle\tilde{n}_{\mathrm{e}} \tilde{V}_{\mathrm{r}}\right\rangle$, where $\tilde{n}_{\mathrm{e}}$ and $\tilde{V}_{\mathrm{r}}$ are fluctuations of the plasma den-

author'se-mail: Eliseev_LG@nrcki.ru

*) This article is based on the presentation at the 26th International Toki Conference (ITC26). sity and radial velocity, \langle\rangle denote time averaging. In its turn, $\tilde{V}_{\mathrm{r}}$ is driven by the $E \times B$ drift, i.e. it is determined by the fluctuating poloidal electric field $E_{\mathrm{pol}}$ and confining toroidal magnetic field $B_{t}: \tilde{V}_{\mathrm{r}}=\tilde{E}_{\mathrm{pol}} / B_{t}$. First estimations of the turbulent particle flux in T-10 were recently performed in $[14,15]$. This report presents new observations of the turbulent particle flux and rotation by HIBP in the T-10 tokamak.

\section{Experimental Setup}

Experiments were performed at the T-10 circular tokamak: major and minor radii $R=1.5 \mathrm{~m}, a=0.3 \mathrm{~m}$, magnetic field $B_{\mathrm{t}}=2.35 \mathrm{~T}$, current $I_{\mathrm{p}}=250 \mathrm{kA}$, so the edge safety factor was $q(a) \sim 2.8$. The power of on-axis electron cyclotron heating $(\mathrm{ECRH})$ was $P_{\mathrm{ECRH}}=1 \mathrm{MW}$.

In T-10, HIBP uses $\mathrm{Tl}^{+}$probing ions with initial energy $E_{\mathrm{b} 1}=200-300 \mathrm{keV}$. HIBP is capable to measure the profiles of local plasma potential $\varphi_{\mathrm{SV}}$ in the Sample volume (SV) from difference between secondary $\left(\mathrm{Tl}^{++}\right) E_{\mathrm{b} 2}$ and primary $\left(\mathrm{Tl}^{+}\right) E_{\mathrm{b} 1}$ energies: $e \varphi_{\mathrm{SV}}=E_{\mathrm{b} 2}-E_{\mathrm{b} 1}$, where $e$ is elementary charge, and the relative density fluctuation $\tilde{n}_{\mathrm{SV}} / n_{\mathrm{SV}}$ from the secondary beam current $\tilde{I}_{\mathrm{tot}} / I_{\mathrm{tot}}$ in the frequency range $<500 \mathrm{kHz}$. The 5 -slit energy analyzer allows us to get the local potentials in several spatial points (SVs) simultaneously. The electric filed is equal to the difference of potentials divided to the distance between slits $j, k: E=\left(\varphi_{\mathrm{Svj}}-\varphi_{\mathrm{SVk}}\right) / d l_{j k}$, where $j, k=1-5$ and $j \neq k$ (Fig. 1).

HIBP scans the plasma cross section by periodical 
variation of beam entrance angle to the plasma (scanning voltage $U_{\text {scan }}$ ) with period $\sim 100 \mathrm{~ms}$. Calculations of beam orbits have shown that HIBP is capable to observe the considerable part of plasma cross-section $0.2<\rho_{\mathrm{SV}}=r_{\mathrm{SV}} / a<$ 1 , however, to retrieve $E_{\mathrm{pol}}$, one should select an appropriate part of detector line, on which SVs are aligned to the same magnetic flux surface (Fig. 2). For the experimental

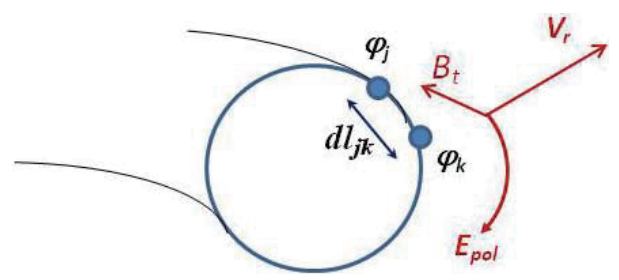

Fig. 1 Outline of the electric field $E_{\mathrm{pol}}$ and radial velocity $V_{\mathrm{r}}$ measurement; $\varphi_{j}$ and $\varphi_{k}$ are potentials in sample volumes on the same flux surface, measured by slits $\# j$ and $\# k$ respectively at a distance $d l_{j k}$
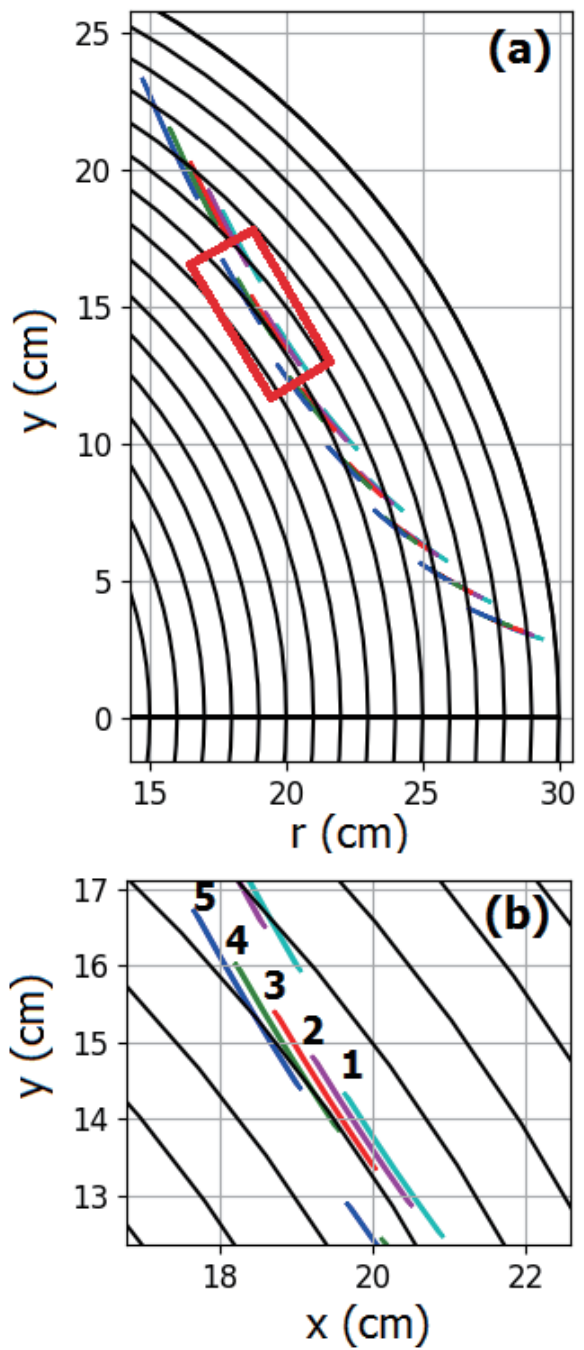

Fig. 2 (a) Detector grid of HIBP with 5-slit analyzer and its part suitable for measurement of poloidal electric field at $\rho_{\mathrm{SV}}=0.8$ (b). Slits are numbered. conditions, shown in Fig. 2 , the selected point $\rho_{\mathrm{SV}}=0.8$ is fitted to measure the turbulent flux. In addition, here the plasma density is not very high $\left(n_{\mathrm{SV}} \approx 1.3 \times 10^{19} \mathrm{~m}^{-3}\right)$, so one can neglect the path integral effect [16] and suppose that the density fluctuation measurement is rather local.

\section{Experimental Results}

The key element in the direct turbulent flux study is simultaneous measurements of plasma potentials $\left(E_{\mathrm{pol}}\right)$ and density at the same spatial point. Correlation analysis allows one to study various parts of frequency power spectra and to analyze the interactions (coherence and cross-phase) between measured plasma parameters. The flux spectral function $\Gamma(f, t)$ may be determined as follows [17]:

$$
\begin{aligned}
& \Gamma(f, t)=\frac{2}{B_{\mathrm{t}}} \operatorname{Re}\left(F_{n_{\mathrm{e}}}(f, t) \cdot F_{E_{\mathrm{pol}}}(f, t)^{*}\right) \\
& =\frac{2}{B_{\mathrm{t}}} \sqrt{\operatorname{coh}\left(n_{\mathrm{e}}, E_{\mathrm{pol}}\right)} \sqrt{\left|\operatorname{PSD}\left(E_{\mathrm{pol}}\right)\right|}\left|\operatorname{PSD}\left(n_{\mathrm{e}}\right)\right| \\
& \quad \cdot \cos \left(\theta\left(n_{\mathrm{e}}, E_{\mathrm{pol}}\right)\right) .
\end{aligned}
$$

Here $F_{n_{\mathrm{e}}}$ and $F_{E_{\mathrm{pol}}}$ are Fourier transforms, $\operatorname{PSD}(x)=$ $F(x) F^{*}(x)$ is the power spectral denisity, for $n_{\mathrm{e}}$ and $E_{\mathrm{pol}}, \theta$ is cross-phase, ${ }^{*}$ means the complex conjugate, $\operatorname{coh}=\gamma^{2}$ is quadratic coherency coefficient between $n_{\mathrm{e}}$ and $E_{\mathrm{pol}}$, Re denotes the real part of the complex function. Below we consider various parts of eq. (1): PSDs for $n_{\mathrm{e}}$ and $E_{\mathrm{pol}}$, cross-phases and coherencies. For brevity, we generally present data obtained from central slits \#2 and \#3. Data from utmost slits \#1, \#4 and \#5 are rather similar.
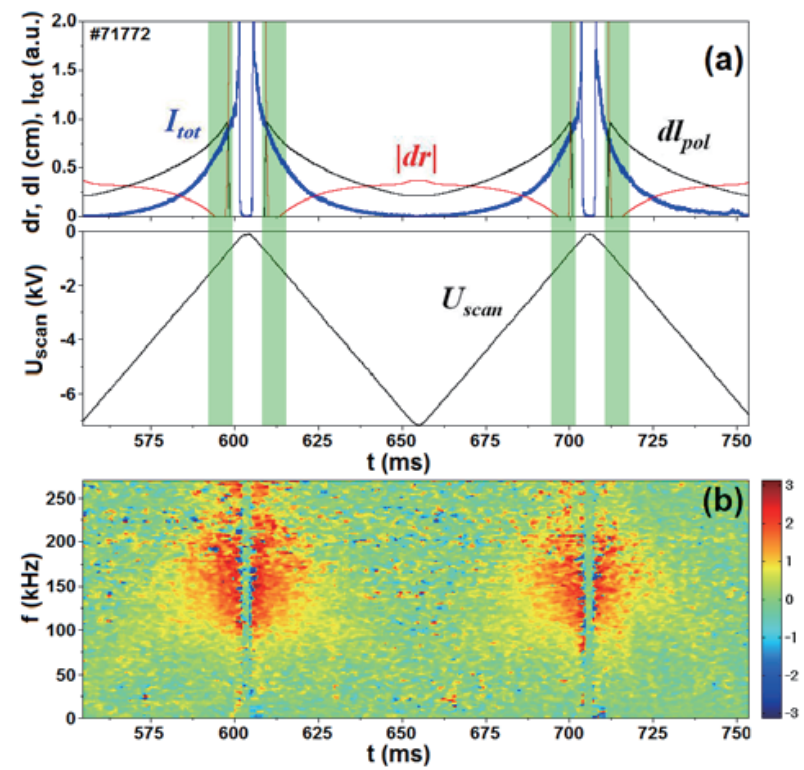

Fig. 3 (a) Variations of beam current $I_{\text {tot }}$, radial and poloidal distances, $d r, d l_{\mathrm{pol}}$, between detector lines for slits \#2 and \#3 during HIBP scan. Vertical green ribbons mark parts of scan, where SVs are separated poloidally, which are applicable for the flux calculation; (b) cross-phases between currents $I_{\mathrm{tot} 2}$ and $I_{\mathrm{tot} 3}$. The most pronounced is quasicoherent mode with a peak frequency $\sim 150 \mathrm{kHz}$. 


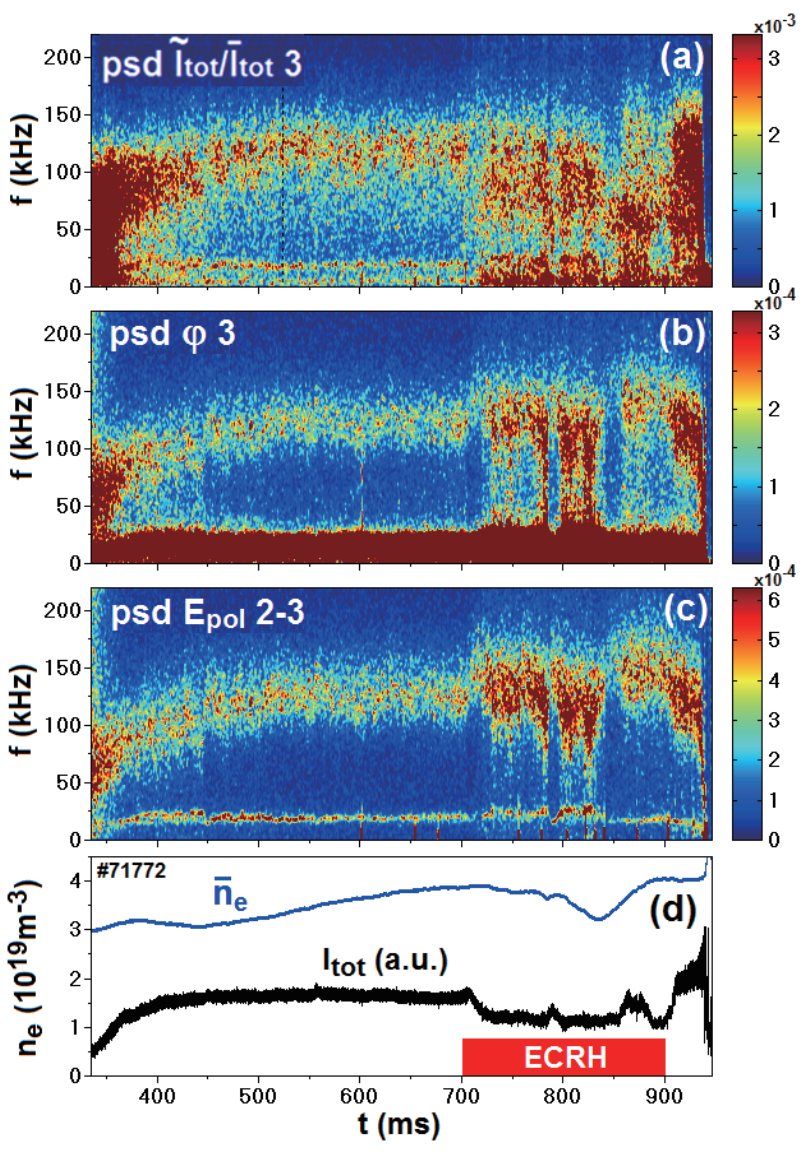

Fig. 4 PSD of fluctuations for density (a), potential (b), and poloidal electric field (c) measured by central slits \#2 and \#3; evolution of the line-average density and beam current $I_{\text {tot }}$ in shot with auxiliary ECRH (d).

Figure 3 (a) shows the evolution of the beam current $I_{\text {tot }}$, the radial $(d r)$ and poloidal $\left(d l_{\mathrm{pol}}\right)$ distances between detector lines for slits \#2 and \#3. Vertical rectangles mark the parts of detector lines aplicable for $E_{\mathrm{pol}}$ definition. Figure 3 (b) shows cross-phases between currents in neighboring slits \#2 and \#3. Finite cross-phase $(\theta<\pi)$ confirms validity of poloidal alignment of SVs.

Figures 2 and 3 show that for $\rho_{\mathrm{Sv}}=0.8, d l_{\mathrm{pol}}<1 \mathrm{~cm}$, poloidal averaging of HIBP is $\sim 1.5-2 \mathrm{~cm}$. The analysis of the cross-phase shows that the typical wavelength of quasicoherent (QC) mode with $f=50-200 \mathrm{kHz}$ is $\lambda_{\mathrm{pol}}=2 \pi d l_{\mathrm{pol}} / \theta=8-3 \mathrm{~cm}$, i.e. the spatial resolution of analyzer is satisfactory to resolve such kind of oscillations. The same way we can estimate the poloidal mode number $m=2 \pi r / \lambda=\theta / d l_{\text {pol }}=20-50$.

Figure 4 presents PSD of fluctuations for density (a), potential (b), poloidal electric field (c) measured by central slits \#2 and \#3. One may see the wide dominating peak of quasicoherent mode $\left(f_{\mathrm{QC}}=50-200 \mathrm{kHz}\right)$ and narrow peak of geodesic acoustic mode (GAM) with $f_{\mathrm{GAM}}=17 \mathrm{kHz}$. ECRH switch-on in the regime under study is accompanied by an increase of the QC fluctuation level and changing its character from quasi steady-state to the bursty one. The

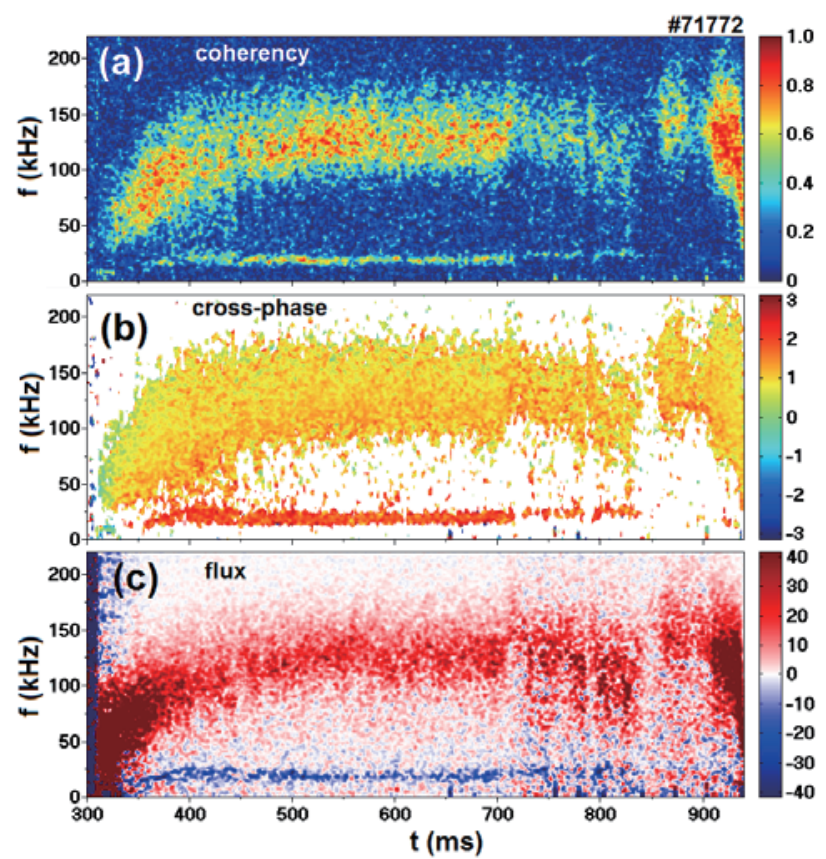

Fig. 5 Coherency $\operatorname{coh}\left(n_{\mathrm{e}}, E_{\mathrm{pol}}\right)$ (a) and cross-phase (b) between fluctuations of density and poloidal electric field $\theta\left(n_{\mathrm{e}}, E_{\mathrm{pol}}\right)$; flux spectral function $\Gamma(f, t)$ measured by central slits \#2 and \#3 (c). Here density fluctuations are evaluated from $\tilde{n}_{\mathrm{e}} / \bar{n}_{\mathrm{e}} \approx \tilde{I}_{\text {tot }} / \bar{I}_{\text {tot }}$.

line-averaged density $\bar{n}_{\mathrm{e}}$ pump-out [18] also takes place, as shown in Fig. 4 (d).

Figure 5 presents coherency (a), cross-phase between $n_{\mathrm{e}}$ and $E_{\text {pol }}$ fluctuations in slits \#2-\#3 (b), and the flux spectral function (c). Similar to potential and density PSDs, presented in Fig. 4, QC mode is dominating in the spectra of Fig. 5. During the Ohmic heating $(\mathrm{OH})$ stage, the coherency for QC mode is rather high, $c o h \leq 0.8$, and the cross-phase $\theta \sim \pi / 3$. Remarkably, during the ECRH stage, the coherency strongly decreases.

Data from Fig. 3 allow us to estimate the poloidal rotation velocity of density turbulence in the frequency range $f=100-250 \mathrm{kHz}$ :

$$
V_{\mathrm{pol}}=\frac{2 \pi d l_{\mathrm{pol}} f}{\theta} \approx 4.5 \mathrm{~km} / \mathrm{s} .
$$

Figure 6 presents the integrated flux in the frequency range $50-200 \mathrm{kHz}$. The fat red line marks the flux $\Gamma_{23}$ (t) obtained by central slits \#2-\#3. One may see that the mean flux increases from $2 \times 10^{19} \mathrm{~m}^{-2} \mathrm{~s}^{-1}$ in $\mathrm{OH}$ stage to $3 \times 10^{19} \mathrm{~m}^{-2} \mathrm{~s}^{-1}$ in ECRH stage of discharge. On top of that in the regime under study the integrated flux is bursty in the ECRH stage, similar to the local potential and $E_{\mathrm{pol}}$, as presented in Fig. 4. Figure 6 shows that the fluxes measured by different pairs of slits are similar, but not equal. The main sources of this difference of fluxes lies in coherence and cross-phases: $\Gamma_{12}$ is higher than others because $\cos \theta_{1-2}$ is higher $\left(\theta_{2-3} \approx \theta_{3-4} \approx \theta_{4-5} \approx 1.0 \mathrm{rad}\right.$, $\left.\theta_{1-2} \approx 0.6 \mathrm{rad}\right) . \Gamma_{45}$ is lower than others because the co- 


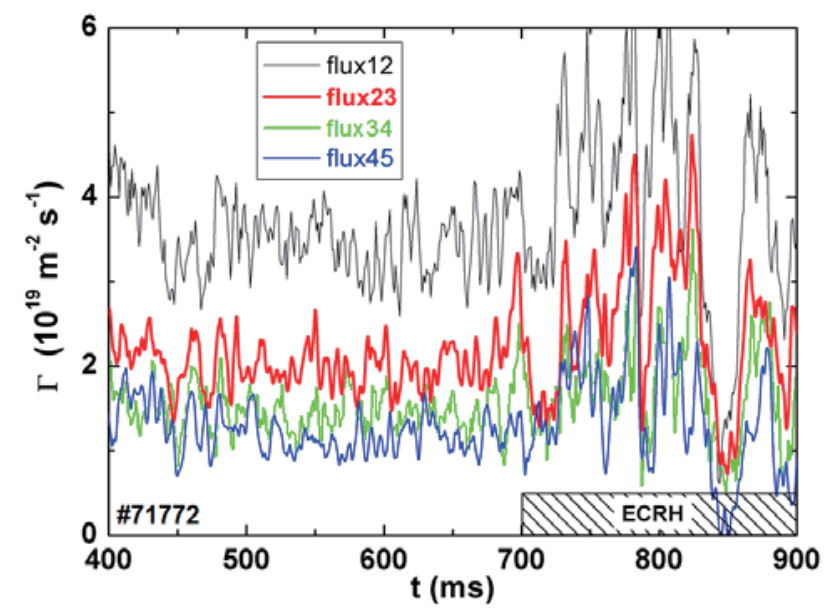

Fig. 6 Frequency integrated flux over the range $50-200 \mathrm{kHz}$ measured by the central (fat red line) and utmost (thin lines) slits.

herency \#4-\#5 is smaller. The increase of turbulent particle flux in ECRH stage is a result of significant increase of amplitude of fluctuations of both $E_{\text {pol }}$ and density $\left(I_{\text {tot }}\right)$ despite of decrease of coherence between them.

\section{Conclusions}

Operation of the multichannel HIBP in the T-10 tokamak have shown internal consistency between simultaneous potential and density measurements by 5 spatial channels. So, it has been shown the capability to measure directly the electrostatic turbulence particle flux in the core of tokamak plasma by HIBP. Initial data shows the out- ward flux in the range of $2 \times 10^{19} \mathrm{~m}^{-2} \mathrm{~s}^{-1}$ at the $\mathrm{OH}$ stage, increasing at the ECRH stage by a factor of 1.5.

\section{Acknowledgment}

Research was funded by Russian Science Foundation, project 14-22-00193.

The work of A.V. Melnikov was partly supported by the Competitiveness Program of NRNU MEPhI.

[1] D.R. Demers et al., Phys. Plasmas 8, 1278 (2001).

[2] Yu.N. Dnestrovskij et al., IEEE Transactions on Plasma Science, (Aug 1994), Vol.22, Issue: 4, pp.310-331.

[3] A.V. Melnikov et al., Nucl. Fusion 57, 072004 (2017).

[4] V.A. Vershkov et al., Nucl. Fusion 55, 063014 (2015).

[5] A.V. Melnikov, Nature Physics 12, 386 (2016).

[6] A.V. Melnikov et al., Plasma Phys. Control. Fusion 48, No 4, S87 (2006).

[7] A.V. Melnikov et al., Nucl. Fusion 55, 063001 (2015).

[8] A.V. Melnikov et al., Nucl. Fusion 57, 115001 (2017).

[9] L.G. Eliseev et al., Study of the large-scale MHD mode and its effect on GAM in the T-10 tokamak, 42nd EPS Conf. on Plasma Phys. P5.159, http://ocs.ciemat.es/ EPS2015PAP/pdf/P5.159.pdf

[10] A.V. Melnikov et al., Nucl. Fusion 54, 123002 (2014).

[11] A.V. Melnikov et al., Nucl. Fusion 56, 076001 (2016).

[12] A.V. Melnikov et al., Nucl. Fusion 56, 112019 (2016).

[13] A.V. Melnikov et al., Nucl. Fusion 52, 123004 (2012).

[14] L.G. Eliseev et al., Probl. Atom. Sci. Techn. Ser. Plasma Physics 107, 241 (2017).

[15] L.G. Eliseev et al., J. Phys.: Conf. Series 907, 012002 (2017).

[16] D.W. Ross et al., Rev. Sci. Instrum. 63, 2232 (1992).

[17] E.J. Powers et al., Nucl. Fusion 14, 749 (1974).

[18] V.F. Andreev et al., Plasma Phys. Control. Fusion 58, 055008 (2016). 